\title{
Habitabilidad, un concepto en crisis. Sobre su redefinición orientada hacia la sostenibilidad
}

\author{
Habitability, a concept in crisis. \\ Its re-definition towards sustainability
}

M. Casals-Tres $^{(*)}$, J. Arcas-Abella ${ }^{(*)}$, A. Pagès-Ramon ${ }^{(*)}$

RESUMEN

La inaplazable respuesta a la crisis ambiental provocada por el sistema productivo industrial, supone el primer y mayor reto que debe afrontar el sector de la edificación, pues conllevará transformaciones profundas en sus objetivos y en sus procedimientos de actuación.

La consecución de la condición de edificación sostenible se aborda en el presente artículo a partir de la aproximación a una redefinición de la misma utilidad social que debe procurar el sector: la habitabilidad. En primer lugar, se analiza la concepción normativa actual y se detectan las barreras que opone frente a las demandas de sostenibilidad. En segundo lugar se exploran alternativas disponibles en el mismo contexto legislativo español que permiten adecuarlo al nuevo paradigma ambiental.

En último término, el artículo también presenta los cambios que la redefinición del concepto de habitabilidad conlleva en la misma dinámica de sector y, concretamente, en la rehabilitación del parque construido.

\section{$113-113$}

Palabras clave: Sector de la edificación, edificación sostenible, habitabilidad, rehabilitación.
SUMMARY

The urgent response to the environmental crisis - produced by our industrial productive system-constitutes the main challenge that the building sector should face, implying profound transformations on its objectives and intervention procedures.

In this article, the possibilities to achieve the sustainability of the building sector are considered from a re-definition of the social utility that the sector should supply: habitability. First, the current regulations are analysed in order to identify the barriers against the sustainability conditions. Secondly, alternatives in the same Spanish regulations contexts are explored to identify the possibilities of adapting these ones to the new sustainable paradigm.

Finally, the article reveals the changes which the redefinition of the concept habitability brings about in the own dynamic of the sector and, specifically, in the building stock renovation.

Keywords: Building sector, sustainable building, habitability, renovation. 


\section{INTRODUCCIÓN}

La constatación de la profunda crisis ambiental global ocasionada por el sistema productivo industrial ha impulsado la reacción social para restringir progresivamente la capacidad emisora de residuos contaminantes que ponen en peligro los sistemas naturales que sustentan la vida. En la actualidad existen compromisos internacionales que limitan alguna de estas emisiones. El caso más destacado es el Protocolo de Kyoto (1997) por ser el primer acuerdo jurídicamente vinculante y por actuar sobre las emisiones de gases de efecto invernadero (GEI) que causan el impacto ambiental más crítico a escala global: el cambio climático. La ratificación de este acuerdo -que en el caso de España significa limitar durante el periodo 2008-2012 el aumento de sus emisiones en un $15 \%$ respecto a las emisiones del año 1990- representa sólo el primer paso de un conjunto de compromisos futuros cada vez más restrictivos, sobre todo para los países desarrollados.

En este marco, la edificación se enfrenta a un grave interrogante entorno a su capacidad para continuar satisfaciendo el compromiso social de generar las condiciones adecuadas para alojar las actividades humanas, dada la dominante tendencia ascendente de generación de residuos contaminantes vinculados al desarrollo de su actividad a lo largo de los últimos años. El cálculo de las emisiones asociadas al sector de la edificación español muestra que en el año 2009 -aunque con un descenso respecto al año anterior- estas emisiones fueron un 58\% superiores a las del año 1990, representando un aumento por encima del promedio de las emisiones españolas, que en el mismo año fueron sólo un $27 \%$ superiores a las del año 1990.

Como con cualquier otro contaminante, la única posibilidad de alcanzar los niveles de emisión de GEl que eviten que las ineludibles restricciones ambientales conlleven una limitación a la capacidad del parque edificado para albergar la población, radica en intervenir en dicho patrimonio para aumentar su eficiencia en el uso de recursos, pues las actuaciones centradas en la nueva edificación -por muy eficientes que sean- no podrán ser nunca condición suficiente para alcanzar dicho grado de optimización si no se interviene sobre el parque construido (1).

Es desde esta perspectiva que la rehabilitación, más que como una solución transitoria orientada principalmente desde la recuperación económica de un sector clave en la economía del país, se revela como una tarea prioritaria que necesariamente deberá estar enfocada desde su vertiente ambiental: el sector de la edificación deberá adoptar a la rehabilitación como principal instrumento continuo de acción para poder garantizar la generación de su utilidad social en un contexto de baja emisividad de GEI. Y, en este sentido, deviene ineludible plantear un nuevo paradigma orientado a alcanzar la condición de sostenibilidad en la edificación.

Por ello, es necesario establecer como punto de partida una definición de edificación sostenible sobre la que sostener un análisis crítico del modelo actual y articular los argumentos del cambio:

La edificación sostenible se puede definir a partir de la transposición de la concepción de desarrollo sostenible institucionalmente aceptada que formuló en 1987 el documento Nuestro Futuro Común -generalmente conocido como Informe Brundtland- (2), como "la edificación que provee la habitabilidad socialmente demandada y opera cerrando los ciclos materiales de todas las actividades implicadas en este proceso" (3).

Proveer habitabilidad -transpuesto de satisfacer necesidades- debe ser entendido como garante de la utilidad social de la edificación, pues en un contexto sostenibilista toda actividad debe orientarse al cumplimiento de la función social que se le otorga. Del mismo modo que sin satisfacer necesidades no tiene sentido el desarrollo, sin habitabilidad no tiene sentido la edificación, por cuanto esta representa su función social básica. Esta condición implica un cambio fundamental: el sector de la edificación deberá abandonar su tradicional función de constructor de edificios para redefinirse como "el conjunto de las actividades destinadas a producir y mantener la habitabilidad necesaria para acoger las actividades sociales" (4).

Cerrar los ciclos materiales -transpuesto de no comprometer a las generaciones futuras-significa que el ritmo de extracción de recursos debe ser igual o menor al de reposición natural y que el ritmo de generación de residuos debe ser igual o menor al de la capacidad de absorción del medio. Las limitaciones de los GEI son el ejemplo más significativo de esta condición. Los objetivos ambientales reconocidos por la comunidad internacional, consistentes en no superar en más de $2^{\circ} \mathrm{C}$ el aumento de la temperatura media global respecto a los niveles preindustriales (5), se traducen en el caso de los países desarrollados en reducciones de entre un 80 y un $95 \%$ de las emisiones del año 2000 para el año 2050 (6). Estas limitaciones resultan profundamente transformadoras para el sector de la edificación español, pues sus emisiones en el periodo 1990-2009 fueron equivalentes al $32 \%$ de las emisiones imputadas a España a lo largo del mismo periodo. 
La magnitud del reto conlleva que las acciones de reducción de emisiones que se planteen sobre cualquier actividad no pueden ser superficiales, temporales y/o esporádicas sino amplias y radicales, y además, en el caso del sector de la edificación, urgentes por el largo ciclo de vida de los edificios.

La improrrogable reconversión del sector de la edificación deberá abordarse, en este sentido, no sólo mediante el desarrollo de estrategias de aumento de eficiencia aplicadas a las viviendas (3), sino a través de cambios estructurales: en primer lugar a partir de la redefinición de su misma utilidad social, es decir, desde la redefinición de la habitabilidad socialmente necesaria que el sector de la edificación deberá procurar bajo los condicionantes de la sostenibilidad (7). En segundo lugar, desde el replanteamiento de los procesos de desarrollo de sus actividades derivado de la redefinición de sus objetivos, a saber, la caracterización de la rehabilitación desde el punto de vista de la edificación sostenible.

En esta línea, el presente artículo propone la aproximación a estos dos ejes mediante el desarrollo de dos planteamientos principales:

El análisis de las barreras que presenta el actual concepto normativo de habitabilidad y la valoración de alternativas contenidas en la misma legislación española que pueden contribuir a la redefinición que demanda la edificación sostenible y su principal instrumento de acción, la intervención en el parque construido.

La presentación de los campos de actuación de la rehabilitación surgidos a raíz de la redefinición de la utilidad que debe producir su actividad

\section{EL ACTUAL MARCO NORMATIVO DE LA HABITABILIDAD}

Actualmente, el concepto de habitabilidad en España se encuentra fundamentado en el conjunto de normativas estatales, autonómicas y municipales destinadas a garantizar el derecho constitucional a disfrutar de una vivienda digna y adecuada, enunciándose como una propiedad aplicable a una edificación que observa una serie de parámetros mínimos dictados por la legislación vigente en cada ámbito geográfico del país. La habitabilidad es, en este sentido, un reconocimiento legal que se articula desde las cualidades que emanan de las condiciones de los espacios y las características materiales de una vivienda.

Esta visión de la habitabilidad se encuentra presente en la mayoría de legislaciones autonómicas vigentes pero tiene su origen en la primera norma estatal aplicable al conjunto del parque construido, la Orden de 29 de febrero de 1.944, por la que se establecen las condiciones higiénicas mínimas que han de reunir todas las viviendas (8).

Desde entonces la normativa de habitabilidad -a través de sus distintas versiones en el tiempo y el espacio- se ha erigido como elemento director de la acción del sector de la edificación, puesto que establece los requisitos indispensables que debe reunir una vivienda para garantizar que es hábil para el alojamiento, es decir, que es apta como morada humana porque dispone de las condiciones socialmente aceptables para ser habitada.

Así ha sucedido en el sector de la nueva construcción, pero en buena medida también en la rehabilitación. Bien lo indica un estudio gubernamental a nivel europeo que señala que la mejora de las condiciones de habitabilidad es, con el 91\% de apoyo, el motivo con mayor consenso para llevar a cabo la rehabilitación de una vivienda (9). Por consiguiente, la actuación de la rehabilitación pasa, en muchos casos, por intervenir en la edificación existente con el fin de restablecer los requerimientos que la normativa pertinente determina. De manera que dicha normativa dirige y enmarca las acciones transformadoras que ejerce la rehabilitación a través de los parámetros de confort, funcionales y constructivos que dicta, y por tanto, actúa como un factor esencial de todo el proceso.

No obstante, como se expone a continuación, el análisis del marco legislativo de la edificación pone de manifiesto que el actual concepto normativo de habitabilidad contiene ciertos planteamientos que pueden representar un importante freno al desarrollo de la edificación sostenible. Unas barreras que no sólo pueden dificultar la extensión del derecho a una adecuada satisfacción de las necesidades de cobijo a toda la población, sino que pueden impedir el desarrollo de las estrategias sostenibilistas vinculadas a la rehabilitación del parque de viviendas existente que exige el escenario actual de reducción de las emisiones de gases de efecto invernadero y de lucha contra el cambio climático.

\subsection{Soluciones materiales como exigencias de la habitabilidad}

En primer lugar, las condiciones de habitabilidad exigidas por las normativas actuales están expresadas sobre soluciones materiales concretas, de manera que se exigen siempre unos determinados bienes con unas características dimensionales, ambientales y constructivas concretas. 
En este sentido, la habitabilidad no se plantea explícitamente desde las necesidades que debería cubrir como respuesta a las demandas sociales; pues en vez de reconocer actividades como el descanso, la higiene o la alimentación, se exigen directamente unas condiciones materiales concretas en forma de espacios-dormitorio, baño, cocina, etc.-, de equipos -muebles, sanitarios, electrodomésticos, etc. - e instalaciones y suministros -electricidad, agua caliente y fría, gas, etc.-

Este planteamiento puede conducir a rigidizar la oferta de modelos de vivienda, mermando la capacidad de responder adecuadamente a los distintos modos de vida hoy en día presentes en nuestra sociedad, de manera que, no sólo puede suponer el incumplimiento del mandato constitucional de extender el derecho a una vivienda digna y adecuada a todos los colectivos, sino que puede impedir alcanzar los objetivos de la edificación sostenible definidos a partir de la transposición de los conceptos expresados en el Informe Brundtland. La no consideración de las necesidades a satisfacer puede conllevar el uso inadecuado de los recursos, ofreciendo modelos con condiciones que, o bien no se ajustan a las demandadas, o bien exceden los requerimientos de sus habitantes.

Aun así, la concreción y rigidez de las condiciones físicas y materiales de la actual normativa de habitabilidad representan para el sector de la nueva construcción objetivos alcanzables, pero para la rehabilitación pueden suponer una manifiesta dificultad.

La acción de rehabilitar, a diferencia de la nueva construcción, implica considerar el patrimonio construido, es decir trabajar a partir de un estado previo de enorme complejidad a múltiples niveles -físicos, legales, económicos, culturales, etc-. En este escenario plagado de particularidades, la actual concepción monolítica de la habitabilidad puede reducir, en gran medida, la capacidad de interpretación -y por tanto de rehabilitación- del parque existente de viviendas y de la habitabilidad que contienen, pues esta capacidad está vinculada a la precisión y versatilidad del enfoque con que se contemplan.

\subsection{La vivienda como unidad de actuación de la habitabilidad}

En segundo lugar, la actual concepción de la habitabilidad que emana de las normativas autonómicas se encuentra circunscrita a la vivienda, debido a que esta constituye la unidad de actuación de la edificación y el sujeto legal constitucionalmente reconocido.

Sin embargo, esta concepción presenta dificultades frente a una nueva articulación sostenible que procure la satisfacción de las necesidades operando en ciclos materiales cerrados, puesto que, aunque la vivienda constituye uno de los espacios que acogen mayor número de necesidades básicas y consume, paralelamente, un mayor volumen de recursos, no es posible dejar de observar que la sostenibilidad de las actividades que alberga no depende únicamente de ella, sino que se encuentra relacionada con cuestiones de escala urbana y territorial. Así, la propia morfología de la vivienda, y el tipo de infraestructuras y flujos que la sirven depende de la estructura urbana y el sistema de gestión, pues no es lo mismo, por ejemplo, un piso con una única fachada a la calle, caldera central de gas natural y servicio de recogida selectivo de basuras puerta a puerta, que una casa unifamiliar aislada, con calefacción por gasóleo y un contenedor de residuos unitario situado a $300 \mathrm{~m}$.

Asimismo, una unidad de actuación ceñida a la vivienda es coherente con la hasta ahora dinámica del sector de la nueva construcción, puesto que se establece desde sus leyes funcionales y constructivas. Toda nueva construcción debe ejecutar todas y cada una de las piezas que forman una vivienda -las habitaciones, la cocina, el baño, etc.- puesto que debe ofrecer al mercado unidades funcionales completas; y al mismo tiempo, fabricar todos los elementos que constituyen la vivienda como artefacto -estructura, cerramientos, instalaciones, etc.- siguiendo las lógicas estructurales y constructivas.

No obstante, cuando se trata de rehabilitar la ciudad existente la vivienda como unidad de actuación puede carecer de sentido, puesto que a nivel funcional y constructivo no siempre es preciso intervenir sobre el conjunto de dicha unidad, sino que puede presentar más ventajas actuar simultáneamente sobre una fracción de un conjunto de viviendas.

En este sentido, la vivienda como unidad única de la actual concepción de la habitabilidad puede devenir una dificultad en el desarrollo de la rehabilitación por varios motivos. Por un lado, puede representar una unidad demasiado extensa, ya que puede forzar a intervenir sobre un conjunto de elementos funcionales y constructivos, obstaculizando la actuación sistemática sobre unidades menores como son las instalaciones, las ventanas o las cubiertas, entre otros. En paralelo, el trabajo centrado en el marco de la vivienda puede impedir la adopción de soluciones que se desarrollen en escalas superiores, a saber, el edificio, la manzana o el barrio. Estrategias que pueden ofrecer una mayor eficiencia, tanto en los procesos de rehabilitación, como en el consumo de recursos y la generación de residuos derivados del uso y mantenimiento de la solución adoptada. 


\section{LA REDEFINICIÓN DE LA HABITABILIDAD DESDE LAS ALTERNATIVAS DIPONIBLES EN EL CONTEXTO NORMATIVO}

Ahora bien, la necesaria superación de las barreras que presenta la concepción normativa actual de habitabilidad frente al desarrollo de la edificación sostenible, puede ser abordada mediante la detección de planteamientos alternativos presentes en la misma legislación española. Por un lado, en las sucesivas normativas que han regulado la habitabilidad a lo largo de los últimos 100 años a nivel estatal y autonómico, y por el otro, en el conjunto de legislaciones vigentes referente al amplio abanico de alojamientos socialmente aceptados en la actualidad.

El análisis diacrónico del conjunto de legislaciones españolas que han definido históricamente el concepto de habitabilidad abarca las sucesivas leyes que han regulado las distintas categorías de viviendas protegidas y las normativas sobre requisitos mínimos de habitabilidad que deben reunir todas las viviendas del parque, incluyendo en este apartado las legislaciones nacionales derogadas y las normativas autonómicas vigentes resultantes del traspaso de competencias (1981).

El estudio sincrónico del conjunto de legislaciones que regulan el abanico de alojamientos disponibles en la sociedad actual, en cambio, nace de la consideración que, aunque la vivienda representa el modelo de habitabilidad más extendido en nuestra sociedad y en el cual residimos la mayor parte de nuestra vida, no se puede obviar que junto a ella existe un amplio conjunto de alojamientos diversos que igualmente satisfacen la demanda social de cobijo, bien sea para ciertos colectivos, bien sea para determinados periodos de tiempo. Dentro de este grupo alternativo a la vivienda se puede identificar fácilmente a residencias, apartamentos, hoteles y pensiones, alberges, campings, refugios, hospitales, internados, monasterios, conventos, faros, cuarteles, prisiones, etc.

Todos ellos representan modelos social y legislativamente aceptados que, aunque legalmente no se les pueda aplicar el término habitabilidad, efectivamente cumplen la misma función social que el parque de viviendas del país - proporcionar cobijo- $y$, en este sentido, forman parte del fondo de espacios habitables disponibles para la sociedad.

En última instancia, las alternativas que pueden ser detectadas en este amplio contexto normativo, no sólo aportan directrices para la redefinición del concepto de habitabilidad, sino que legitiman este cambio de plantea- miento: en primer lugar porque el hecho de que todas pertenecen a normativas españolas significa que las mismas administraciones del país disponen de criterios flexibles y admiten pluralidad de concepciones de la habitabilidad. En segundo lugar porque el análisis histórico de la normativa de vivienda desvela que el concepto de habitabilidad se ha ido transformando a lo largo de los años $y$, en consecuencia, es factible contemplar un estadio más dentro de su evolución.

\subsection{Soluciones enunciadas desde las personas y sus necesidades}

La revisión del panorama legislativo español revela que, frente al dictado de soluciones materiales concretas como garantía de habitabilidad en la vivienda presente en la normativa vigente, se pueden encontrar enunciados que se inician desde el reconocimiento de las necesidades de las personas.

En este sentido, tanto en las legislaciones de vivienda actuales y históricas, como las relacionadas con otros tipos de alojamientos, se pueden detectar estrategias que, o bien consideran una amplia variedad de perfiles de demanda -proporcionando soluciones habitacionales específicas-, o bien proponen mecanismos que contribuyen a ofrecer condiciones de habitabilidad diversas, condiciones capaces de ajustarse a las distintas configuraciones de necesidades presentes en nuestra sociedad.

Las principales aportaciones de los planteamientos legislativos analizados se pueden enunciar bajo cuatro secciones:

\section{Modelos habitacionales adaptados a distintos perfiles de demanda}

En primer lugar, el análisis diacrónico del conjunto de legislaciones españolas que han definido históricamente el concepto de habitabilidad permite detectar que, a diferencia de la legislación vigente, existen documentos que reconocen múltiples perfiles de demanda.

Las normativas de vivienda social vigentes hasta finales de los años 60 recogen distintas modalidades de hogares que, más allá de la familia nuclear, consideran desde personas solas o trabajadores en tránsito hasta familias reducidas, numerosas o en proceso de crecimiento. Un ejemplo de ello lo constituye el Reglamento provisional de la Ley de Casas Baratas de 1921 (10):

"En el grupo de casas colectivas se entienden incluidas las casas con varios cuartos para ser alquilados a diferentes familias; las construc- 
1. Superficie de la vivienda según número de personas ocupantes regulada por la normativa de habitabilidad vigente en La Rioja, en Canarias y en Cataluña.

2. Número de categorías que establece la normativa vigente de establecimientos de alojamientos turísticos y vivienda en Cataluña. ciones destinadas a proporcionar albergue a trabajadores de tránsito; las destinadas, en las grandes ciudades, al alojamiento de personas solas que no las utilicen más que para dormir (casas de dormir), y, en general, todas aquellas edificaciones que (...) puedan asimilarse a las comprendidas a los casos anteriores".

\section{Pluralidad de condiciones de habitabilidad}

En segundo lugar, dentro del actual conjunto de normativas autonómicas de habitabilidad se reconocen notables variaciones en los valores de los parámetros que caracterizan las soluciones materiales de cada alojamiento. Aunque todas ellas persiguen el objetivo común de proporcionar una vivienda que reúna las condiciones de habitabilidad demandadas por la sociedad española, y coincidan en determinar unas mismas soluciones materiales, se puede observar importantes divergencias en las condiciones mínimas de estas, tanto con relación a las características dimensionales y ambientales de los espacios, como en los bienes y flujos materiales que los equipan.

Así, los dormitorios, cocinas o equipamientos de la vivienda pueden ser bien distintos en función de la Comunidad Autónoma que los regule, si bien cada uno de ellos puede considerarse habitable para una misma función. Si se extiende la comparación al conjunto de funciones que acoge una vivienda en cada uno de estos territorios se observa que existe cierta flexibilidad legislativa en cuanto cuáles son las condiciones materiales mínimas que garantizan la habitabilidad.

Tres casos significativos que conforman, en relación con uno de los parámetros esenciales de la concepción actual de la habitabilidad -la superficie de la vivienda-, los extremos de la envolvente normativa trazada por el conjunto de legislaciones vigentes, los constituyen las condiciones de habitabilidad de La Rioja (11), Canarias (12) y Catalunya (13) (Figura 1).

\section{Multiplicidad de grados de habitabilidad}

En tercer lugar, la observación del conjunto de alojamientos disponibles en España alternativos a la vivienda, permite detectar distintos grados de habitabilidad expresados en categorías, de manera que, en lugar de disponer tan sólo una única conformación de la habitabilidad -como actualmente considera la normativa de vivienda-, se establecen múltiples niveles de habitabilidad en función de la amplitud de las prestaciones que ofrece un espacio. Los incrementos de habitabilidad que suponen el ascenso de categoría se obtienen mediante la mejora de las condiciones espaciales -principalmente mayor superficie útil-, el aumento del número y calidad de los equipos, la ampliación de los flujos materiales, y la multiplicación de los servicios ofrecidos al habitante. En general, a mayor nivel de habitabilidad más demandas son cubiertas y en mayor grado.

Esta concepción progresiva de la habitabilidad fue iniciada por las primeras normativas de establecimientos turísticos en España y actualmente continúa vigente y plenamente aceptada social y legislativamente en la mayoría de alojamientos turísticos de nuestro país, llegando a distinguir hasta 7 categorías de ofertas de habitabilidad (14) frente a la categoría única de la normativa de vivienda actual (Figura 2).

\section{Soluciones habitables basadas en servicios}

En cuarto lugar, entre los alojamientos socialmente admitidos se identifican diversas soluciones habitables basadas en servicios en lugar de ser expresadas sobre condiciones materiales, de modo que en lugar de especificar directamente y sin margen de variación las características de unos espacios, bienes e infraestructuras, se reconoce de forma genérica la necesidad de poder solventar unas funciones, dejando abiertas sus posibles soluciones materiales. Así, la concreción y rigidez de las condiciones físicas y materiales de la
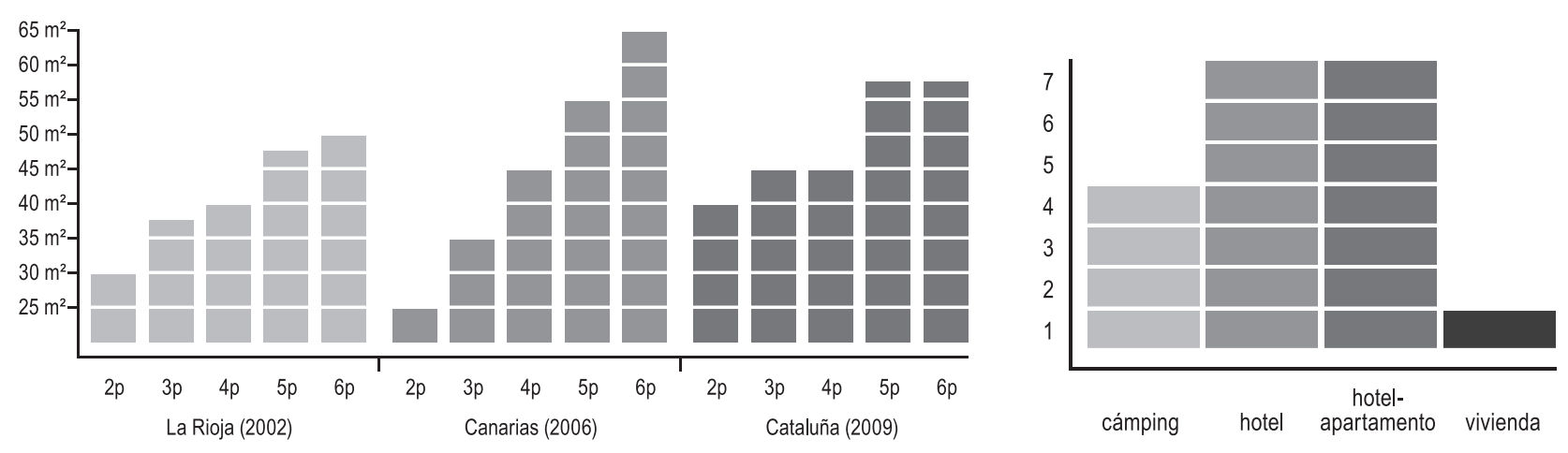
actual normativa de la vivienda, contrasta fuertemente con el enunciado exigencial de la mayoría de las legislaciones de alojamientos que se limitan a especificar la presencia de determinados servicios. Servicios que, en muchos casos, no son anexos sino que son considerados básicos para la habitabilidad, como son la alimentación -resuelta en los establecimientos hoteleros-, la limpieza de la ropa -contemplado en el camping- o el mantenimiento del propio espacio habitable -considerado en la mayoría de establecimientos turísticos-.

Entre todos los alojamientos expuestos el camping aporta la mejor muestra de la capacidad de la legislación de establecer las condiciones de habitabilidad mediante la especificación de servicios. Para un establecimiento de máxima categoría, la primera normativa catalana aprobada en 1986 (15) señalaba:

"Servicio de asistencia médica con visita diaria y servicio asegurado de ATS o enfermero. Servicio telefónico. Servicio de lavandería y plancha. Servicio de lavado de coches. Servicio de vigilancia diurna y nocturna. Servicio de custodia de valores con caja fuerte. Servicio de recogida y distribución diaria de correspondencia. Servicio de camping-gas".

El estudio del panorama legislativo español proporciona un completo abanico de estrategias de conformación de la habitabilidad que, en lugar de garantizar su disponibilidad mediante la disposición de unas soluciones materiales concretas, se articulan a partir del reconocimiento de las necesidades de las personas y de la amplia variedad de situaciones -resultado de la edad, sexo, condición social y cultural, renta, entorno, etc.- que exigen respuestas concretas derivadas de esta especificidad.

En este sentido, tanto la contemplación de un amplio espectro de perfiles como la diversificación de modelos habitacionales a través de grados o el enunciado a través de servicios, posibilitan la apertura del actual concepto de habitabilidad implícito en la vigente normativa de vivienda, proporcionando la diversidad de condiciones de habitabilidad que hoy en día demanda nuestra sociedad.

Este planteamiento, autorizado por la propia legislación, enlaza directamente con el primer objetivo de la edificación sostenible que emana de la definición del Informe Brundtland, satisfacer las necesidades, y, en cierta medida contribuye también al segundo, por cuanto los recursos serán utilizados para fines precisos. Y de este modo, permite encauzar la acción del sector de la edificación, la rehabilitación del parque de viviendas, hacia la condición de sostenibilidad:

Por un lado, porque una habitabilidad enunciada desde las personas y sus necesidades fomentaría una rehabilitación orientada a proporcionar unas condiciones de habitabilidad plurales y diversificadas, más ajustadas a las demandas sociales $y$, por tanto, sin un uso excesivo o inadecuado de los recursos. En este sentido, el reconocimiento de la variedad de modos de vidas debería funcionar como una herramienta de optimización de los recursos invertidos en cada proceso de rehabilitación.

Por el otro, porque el desarrollo de una visión normativa de la habitabilidad más flexible debería permitir una lectura más atenta, más precisa, y, por lo tanto, más efectiva del patrimonio existente que agilizara y optimizara esta urgente actividad que supone la rehabilitación en la consecución de los retos medioambientales hoy ineludibles para nuestra sociedad. Debería ser posible, en este sentido, que la legislación, en lugar de aceptar que ciertas viviendas existentes no disponen de las condiciones mínimas de habitabilidad, fuera capaz de reconocer qué grado de habitabilidad ofrecen o pueden ofrecer una vez rehabilitadas y dotadas de servicios y equipamientos, y en consecuencia, estableciera para quién, o en qué situación, son habitables. Porque, en definitiva, la habitabilidad debería expresarse desde las necesidades de las personas antes que desde las propiedades de las viviendas.

\subsection{La diversidad de escalas de actuación de la habitabilidad}

Asimismo, la lectura del compendio legislativo vinculado al concepto de habitabilidad permite mostrar que, frente a la actual concepción que adopta la vivienda como unidad de actuación, han existido opciones normativas que abarcaban otras escalas.

La observación de las legislaciones del conjunto de alojamientos presentes en nuestra sociedad y de la evolución de la normativa de vivienda desvela estrategias de configuración de la habitabilidad que actúan en ámbitos que exceden la vivienda, desde el inmueble hasta la ciudad; estrategias que permiten dar respuesta al conjunto real de necesidades -las propias de la vida urbana-y operar en la escala adecuada para gestionar la dinámica material.

Las contribuciones procedentes de la revisión del marco legal estudiado se pueden sintetizar bajo dos secciones: 
3. Nivel de privacidad de las funciones de dormir (d), relación social (r) comer (c) y higiene ( $h$ ) que establece la normativa vigente de establecimientos de alojamientos turísticos y vivienda en Cataluña.

4. Aspectos regulados por cada normativa española (1912-1978) y catalana (1983-2009) que ha establecido condiciones de habitabilidad.

\section{Extensión de la habitabilidad a la escala del} inmueble

El análisis de la pluralidad de alojamientos socialmente admitidos paralelos a la vivienda permite advertir mecanismos de conformación de las condiciones de habitabilidad que se fundamentan a la escala del inmueble. Residencias, establecimientos turísticos y religiosos, cuarteles, prisiones, entre otros, son ejemplos evidentes de modelos de habitabilidad que, contrariamente a la normativa de vivienda vigente que sitúa todas las funciones dentro del ámbito doméstico, desplazan ciertas actividades a una escala menos privada donde las condiciones que permiten satisfacer una necesidad son compartidas por colectivos mayores. Así, frente al funcionamiento de la casa, prácticamente todos los alojamientos mencionados incorporan espacios donde las actividades son resueltas en común, es decir con personas que no necesariamente forman parte del grupo más próximo.

A continuación (Figura 3) se muestran las diferentes opciones de organización de las principales funciones que debe solventar la habitabilidad que contemplan las normativas de los distintos alojamientos turísticos y la normativa de vivienda, como muestra especialmente significativa de la amplitud de planteamientos vigentes en nuestro país. Sin embargo, las aportaciones de estos aloja- mientos no sólo se sostienen en la posibilidad de revisar la escala de satisfacción de las necesidades implícitas en la normativa de habitabilidad, sino que también se formulan a partir del amplio espectro de necesidades que pueden contemplar al gestionar de forma integrada conjuntos mayores a los de la vivienda. La recientemente aprobada normativa de establecimientos hoteleros en Cataluña (14) reconoce, en este sentido, la posibilidad de alcanzar baremos de calidad superior en función de la disponibilidad de servicios de gimnasio o piscina, estética personal, prensa, atención médica, o guardería.

El desplazamiento de ciertas funciones a nivel de inmueble o de incorporación de nuevos servicios, deberá ser precedido por la flexibilización de la actual concepción legal de la propiedad, así como por un cambio de visión de los habitantes hacia los límites y la posición de los espacios disponibles para el desarrollo de sus actividades dentro del inmueble. En esta dirección se puede encontrar el ejemplo de la vigente normativa catalana que elimina la obligatoriedad de que la vivienda constituya un espacio continuo, permitiendo la existencia de espacios disociados del núcleo habitacional principal que facilitarían la adaptación del domicilio a la variabilidad actual de las estructuras familiares y sus periodos de transición.
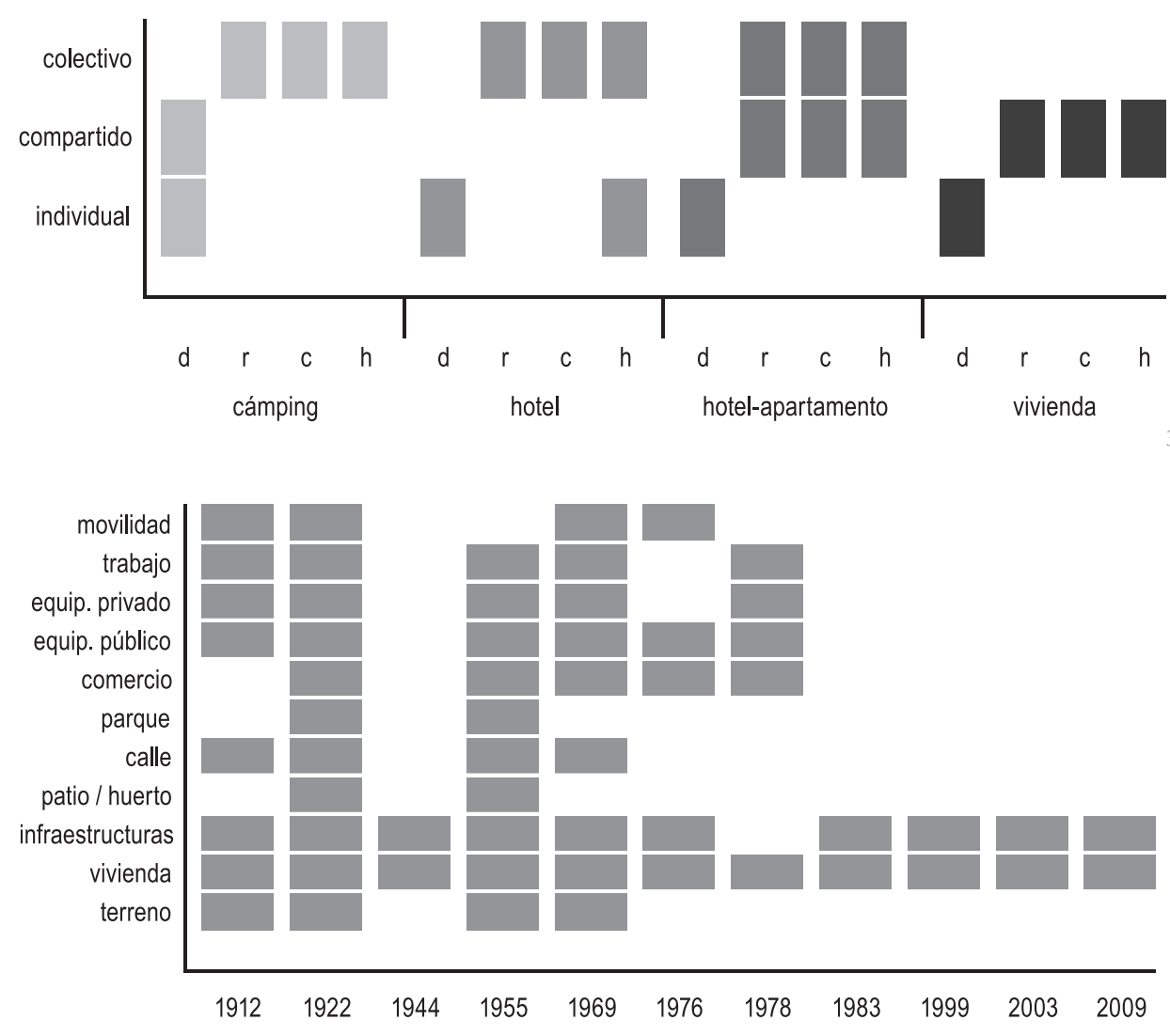


\section{Evolución histórica de la escala de la habitabilidad}

A partir del análisis diacrónico del conjunto de legislaciones españolas que han definido históricamente el concepto de habitabilidad se pueden distinguir múltiples opciones de extensión del concepto de habitabilidad. Desde las que regulan únicamente condiciones de la vivienda, hasta las que sobrepasan esta escala para incluir exigencias sobre elementos propios del ámbito urbano, como son la calle, los servicios públicos, los comercios, etc.

Las normativas de habitabilidad que contemplaban un mayor número de escalas coinciden con las legislaciones relacionadas con las primeras categorías de viviendas protegidas: Casas Baratas (1912 y 1922) (10), Viviendas de Renta Limitada (1955) (16), Viviendas de Protección Oficial (1969) (17). En todas ellas, se explicita una concepción de la habitabilidad que vincula vivienda, entorno urbano y equipamientos y servicios, proporcionando indicaciones relativas a la presencia y características del emplazamiento, la vivienda, la calle, los patios y huertos, los comercios, los servicios públicos y privados -escuela, mercado, iglesia, campos deportivos, lugares de reunión, espectáculos, parques y jardines- o los medios de transporte.

Como muestra especialmente significativa de esta concepción amplia de la habitabilidad se puede citar un fragmento perteneciente al Reglamento provisional de la Ley de Casas Baratas de 1921 (10):

"Se considerarán como parte integrante de las casas baratas los patios, huertos y parques y los locales destinados a gimnasio, baños, escuelas y cooperativas de consumo, que sean accesorios de una casa o grupo de casas baratas y guarden con ella la debida proporción en cuanto a su extensión e importancia".

Paralelamente, se detecta la presencia de otro conjunto de legislaciones formado por las Viviendas sociales (1976) (18) y las Viviendas de Protección Oficial (1981) (19) que, junto a las especificaciones sobre la vivienda y sus infraestructuras, exigen la presencia de determinados servicios urbanos ubicados a unas distancias máximas de la vivienda. En estas normativas, como muestra el fragmento de la ley deViviendas Sociales de 1976 expuesto a continuación, la habitabilidad adquiere una dimensión urbana excediendo la escala de la vivienda para involucrar otras funciones relacionadas con la vida urbana como son el comercio, el ocio, la educación, la sanidad, el trabajo o el transporte:

"Para que las familias que habiten en las Viviendas Sociales, puedan satisfacer de modo adecuado sus necesidades primarias de carácter social y comunitario, se establece como obligatoria para toda la promoción de Vivienda Social, la disponibilidad de equipamiento mínimo (...), situado a distancias no superiores de las que figuran en el siguiente cuadro [Tabla 1]."

Tabla 1

Equipamiento mínimo y distancias máximas según la Ley de Viviendas Sociales (1976)

\begin{tabular}{|c|c|}
\hline Equipamiento social y comunitario & $\begin{array}{c}\text { Distancia } \\
\text { máxima } \\
\text { en m }\end{array}$ \\
\hline Comercio de uso diario . . . . . . . . . . & $\leq 250$ \\
\hline Guardería infantil $\ldots \ldots \ldots \ldots \ldots$ & $\leq 500$ \\
\hline Centro de Educación General Básica . & $\leq 1.000$ \\
\hline Aparcamiento o Garaje-aparcamiento . & $\leq 250$ \\
\hline Parada de transporte colectivo ...... & $\leq 250$ \\
\hline
\end{tabular}

Fuente: (18).

Desde esta perspectiva, se puede elaborar un cuadro entorno a la diversidad de escalas del concepto de habitabilidad presente en la historia de la legislación española, donde se recogen los aspectos regulados por cada una de las normativas que establecen o han establecido las características precisas para un adecuado alojamiento humano (Figura 4).

El análisis diacrónico de la legislación pone de manifiesto que el actual enfoque de la habitabilidad, centrado básicamente en la vivienda, es el resultado de un proceso histórico de reducción de la escala del concepto de habitabilidad, es decir, de pérdida progresiva de aspectos del ámbito urbano que originariamente se consideraban imprescindibles para un alojamiento adecuado.

En este sentido, la limitación de la habitabilidad al ámbito doméstico ha ido acompañada de un proceso de cesión de competencias a otras normativas, a saber, normativas técnicas -CTE, RITE, etc.- planeamiento urbano y planeamiento territorial. Generando en consecuencia, un enfoque segregado que aborda cada escala desde múltiples normativas dictadas por distintas administraciones y desde escalas espaciales y geográficas muy diferentes, haciendo realmente difícil valorar los recursos precisos para proveer finalmente lo que se considera una habitabilidad socialmente aceptable, que debe incluir el acceso a los servicios públicos en tanto éstos lo son por cuanto socialmente se considera que deben estar al alcance de todos.

Finalmente, el análisis histórico de la legislación de la habitabilidad y del conjunto de alojamientos actuales revela que, en contraposición al enfoque vigente de la vivienda, 
han existido normativas que han considerado la dimensión urbana de la habitabilidad.

La visión aportada por estas legislaciones, que integra bajo una misma perspectiva el conjunto de necesidades básicas socialmente reconocidas y los elementos precisos para su satisfacción -desde la escala doméstica hasta la urbana-, puede representar una alternativa que contribuya a la redefinición de la habitabilidad exigida por la necesaria reconversión del sector de la edificación hacia la sostenibilidad.

En primera instancia, la adopción de la escala urbana debería permitir dotar a la habitabilidad de la dimensión socialmente exigible; igualmente que no se puede admitir una vivienda sin condiciones para el descanso o la higiene, tampoco debería ser aceptable hoy en día que los ciudadanos no tengan garantizado el acceso físico a servicios públicos básicos para una ciudadanía digna, como la educación, la sanidad, la cultura o el deporte.

En segunda instancia, la extensión de la unidad de acción de la edificación hasta el ámbito municipal debería posibilitar la generación de una visión integral coherente con la sostenibilidad, pues a nivel urbano es donde se manifiestan las necesidades sociales y donde se toman las decisiones que determinan los recursos precisos para satisfacerlas. Claro está que la forma urbana, las infraestructuras, la organización de los equipamientos y espacios públicos en la ciudad, o los sistemas de movilidad tienen un papel crucial en la habitabilidad de un municipio, tanto en las condiciones de las mismas viviendas, como en la accesibilidad a los servicios urbanos.

En tercera instancia, una habitabilidad planteada a escala urbana debería proporcionar a la rehabilitación de la dimensión y de los instrumentos que requiere para devenir el motor de transformación del parque construido hacia un modelo de baja emisividad:

Por un lado, porque la rehabilitación a escala urbana puede permitir actuaciones sistemáticas sobre elementos funcionales o constructivos concretos del parque existente que hagan posible el desarrollo de métodos de intervención altamente eficientes. La ciudad podría ser leída, desde este punto vista, no como una suma de viviendas, sino, por ejemplo, como miles de metros de fachadas, de cubiertas, de demandas de climatización o de instalaciones proveedoras de servicios que pueden ser intervenidos sistemáticamente.

Por el otro, porque la consideración de la dimensión urbana, a diferencia del enfoque centrado en la vivienda, puede permitir el desarrollo de estrategias de rehabilitación que contemplen la dotación de habitabilidad a escalas más adecuadas y óptimas desde la perspectiva social y ambiental. A nivel de recursos, tiempo hace que se ha demostrado que la intervención sobre la manzana o el barrio puede posibilitar la implantación de sistemas energéticos de elevada eficiencia, como es, por ejemplo, el district heating o la cogeneración. A nivel funcional, soluciones basadas en equipamientos colectivos que sirven a decenas o centenares de viviendas pueden facilitar servicios y acoger actividades imposibles de solventar dentro de cada uno de los espacios domésticos.

\section{LA REHABILITACIÓN DESDE LA PERSPECTIVA DE LA EDIFICACIÓN SOSTENIBLE}

La aproximación a una nueva concepción de la habitabilidad a partir de los planteamientos expuestos supone un importante paso hacia la consecución de la condición de edificación sostenible, entendida como aquella que provee la habitabilidad socialmente necesaria operando en ciclos materiales cerrados.

No obstante, la reconversión del sector de la edificación exigida por el proceso de descarbonización de la economía no se puede circunscribir sólo a la reformulación de su marco conceptual, sino que requiere inevitablemente la revisión de los procedimientos de actuación del sector.

La rehabilitación constituye, como se ha expuesto, el instrumento de acción que debe adoptar el sector de la edificación, pues el parque construido es la principal fuente de emisiones de $\mathrm{GEI}$, pero previamente es necesario verificar su enfoque para con los objetivos del sector fijados bajo la óptica de la sostenibilidad.

Actualmente la rehabilitación de edificios se define a nivel europeo como "el conjunto de actuaciones necesarias para mejorar las condiciones estructurales del edificio, la eficiencia energética, la protección del medio ambiente, la utilización de energías renovables, y los aspectos arquitectónicos y para garantizar su seguridad y estanquidad" (9), es decir, como una intervención puntual sobre las características materiales de un edificio en vistas a incrementar sus prestaciones. Este proceder es característico del sector de la edificación en su planteamiento actual, es propio de la nueva construcción: el edificio es el producto resultante de su actividad, bien sea a partir de la creación ex novo, bien sea a través de la actuación en construcciones existentes mediante su rehabilitación física. 
La articulación de una práctica rehabilitadora alineada con los principios de la edificación sostenible, en contraposición a su enfoque actual, debería tomar como sujeto al modelo de habitabilidad real, y en consecuencia iniciarse desde el reconocimiento e intervención en los tres factores que caracterizan la satisfacción de la necesidad social de albergue de las actividades humanas a escala urbana: el parque edificado, la ocupación por los habitantes, y los flujos de recursos y residuos implicados en esa ocupación.

El parque edificado es el conjunto de recursos materiales sólidamente organizados que conforman las unidades de alojamiento de actividades y las infraestructuras que las ordenan, conectan y abastecen. Aunque su reconocimiento ya forma parte integrante de la visión actual del sector de la edificación debe ser abordado desde la rehabilitación considerando su complejidad a escala urbana y territorial, y las posibilidades que alberga para proporcionar la habitabilidad socialmente demandada.

La ocupación por los habitantes supone el complejo de personas con necesidades cambiantes derivadas de situaciones evolutivas específicas -edad, sexo, condición social y cultural, renta, entorno, etc.- estructuradas en núcleos y redes familiares y/o sociales distribuidas en el parque construido. Su apreciación debe ser incorporada a la acción rehabilitadora debido a que son los demandantes de las utilidades que debe procurar el sector y los agentes que finalmente, como consecuencia del uso del espacio, determinan el consumo de recursos.

Los flujos de recursos y residuos son la multiplicidad de materiales con baja entropía captados del medio y posteriormente desorganizados en el proceso de consumo de su utilidad. Su consideración es clave para el sector de la edificación ya que será el elemento limitador del modelo de habitabilidad.

El conjugado de estos tres factores tiene que devenir el campo de acción de la rehabilitación en cuanto instrumento del sector de la edificación, pues una intervención parcial no será capaz de garantizar el cumplimiento de los objetivos de reducción de las emisiones de GEI. No obstante, la consideración de los factores mencionados no puede articularse sólo desde el ámbito competencial del sector, sino que requiere cambios en otras esferas. En primer lugar, es necesario formular metodologías de análisis y documentación de la realidad que permitan recabar datos precisos y actualizados de los tres campos, advirtiendo las correlaciones existentes entre ellos. Sólo desde este conocimiento es posible operar sobre el modelo de habitabilidad.
En segundo lugar, es indispensable revisar o armar nuevas reglamentaciones que asimilen la complejidad de la rehabilitación inherente a una visión global del parque edificado, los habitantes y los flujos de recursos y residuos. Los acercamientos del CTE a la rehabilitación (20) y la inspección técnica de edificios, en este sentido, constituyen un primer abordaje a nivel técnico que debe ser reconsiderado para organizar un marco legislativo que entienda que rehabilitar no consiste en intervenir en un momento dado en un edificio, sino gestionar el modelo de habitabilidad a lo largo del tiempo en un escenario de bajo impacto ambiental.

\section{CONCLUSIONES}

El reto que supone la grave crisis ambiental ocasionada por el sistema productivo industrial, debe ser abordado desde todos los sectores de la sociedad, y, en el caso español, especialmente desde la edificación, por cuanto su actividad equivale a un tercio de las emisiones de GEl del país. La coyuntura económica actual ofrece, en este sentido, una oportunidad para instaurar una nueva dinámica sostenibilista basada en la rehabilitación, una oportunidad que debe ser aprovechada para articular un nuevo modelo sustentado desde:

Un sector de la edificación orientado a proveer la habitabilidad socialmente demandada y que opere cerrando los ciclos materiales de los recursos implicados en su actividad.

Una definición de la habitabilidad que, superando las barreras que impone su concepción normativa actual, se enuncie desde el reconocimiento de las personas y sus necesidades, y que adopte la escala urbana como ámbito de actuación.

Una rehabilitación que, contrariamente al enfoque actual centrado sólo en la dimensión material, integre estrategias en todos los campos del modelo de habitabilidad real: el parque edificado, la ocupación de ese parque por los habitantes, y los flujos de recursos y residuos.

Un programa de exploración y documentación de la realidad capaz de dotar del nivel de conocimiento que requiere la intervención en el modelo de habitabilidad.

Un marco legislativo coherente con los objetivos y procedimientos expuestos para el sector de la edificación, que impulse los cambios necesarios para alcanzar un paradigma sostenibilista. 


\section{BIBLIOGRAFÍA}

(1) Cuchí, A.; Pagès, A.: Sobre una estrategia para dirigir al sector de la edificación hacia la eficiencia en la emisión de gases de efecto invernadero, p. 143, Ministerio de la Vivienda, Madrid, 2008.

(2) Comisión Mundial sobre el Medio Ambiente y el Desarrollo: Nuestro futuro común, p. 460, Alianza Editorial, Madrid, 1989.

(3) Arcas-Abella, J.; Pagès-Ramon, A.; Casals-Tres, M.: "El futuro del hábitat: Repensando la habitabilidad desde la sostenibilidad. El caso español". Revista INVI, n 26 (72), pp. 65-93 (2011).

(4) Cuchí, A.; Wadel, G.; Rivas, P.: Cambio Global España, 2020/50: Sector edificación: la imprescindible reconversión del sector frente al reto de la sostenibilidad, p. 248, Fundación General de la Universidad Complutense. Madrid, 2010.

(5) Naciones Unidas: Outcome of the work of the Ad Hoc Working Group on long-term Cooperative Action under the Convention. Advance unedited version. Draft decision / CP.16. [en línea]. Cancún: The United Nations Climate Change Conference in Cancun, COP 16 / CMP 6, 2010. p. 29. [Fecha de consulta: 2 marzo 2011]. Disponible en: http:// unfccc.int/files/meetings/cop_16/application/pdf/cop16_lca.pdf.

(6) Baker, T.; et al.: Resumen Técnico. El Cambio Climático 2007: Mitigación. Contribución del Grupo de Trabajo III al Cuarto Informe de Evaluación del Panel Intergubernamental de Expertos sobre Cambio Climático, p. 96, Cambridge University Press. Cambridge (Reino Unido) y Nueva York (Estados Unidos de América), 2007.

(7) Naredo, J. M.; Cuchí, A.: "El libro verde de medio ambiente urbano en el ámbito de la edificación". En: Libro verde de medio ambiente urbano, pp. 88-112. Ministerio de Medio Ambiente. Madrid, 2007.

(8) España: "Orden de 29 de febrero de 1.944, por la que se establecen las condiciones higiénicas mínimas que han de reunir las viviendas". Boletín Oficial del Estado, no 61 (1944).

(9) Ministerio de Vivienda: La Rehabilitación del Parque Residencial Existente en la Unión Europea y Otros países Europeos. XVIII Reunión informal de Ministros responsables de Vivienda de la Unión Europea, p. 344, Ministerio de Vivienda, Toledo, 2010.

(10) España: "Real Decreto de 15 de abril, aprobatorio del reglamento de la ley de 12 de junio de 1911 de Casa Baratas". Gaceta de Madrid, n 106 (1912).

España: "Real Decreto aprobando el Reglamento provisional, que se inserta, para la aplicación de la ley de Casas baratas de 10 de Diciembre de 1921". Gaceta de Madrid, no 209 (1922).

(11) La Rioja, España: “Decreto 51/2002, de 4 de octubre, por el que se regulan las condiciones mínimas de habitabilidad que deben reunir las viviendas en el ámbito de la Comunidad Autónoma de La Rioja, así como la concesión y control de las cédulas de habitabilidad". Boletín Oficial de La Rioja, no $132(2002)$.

(12) Canarias, España: "Decreto 117/2006, de 1 de agosto, por el que se regulan las condiciones de habitabilidad de las viviendas y el procedimiento para la obtención de la cédula de habitabilidad". Boletín Oficial de Canarias, n 161 (2006).

(13) Cataluña, España: “Decreto 55/2009, de 7 de abril, sobre las condiciones de habitabilidad de las viviendas y la cédula de habitabilidad". Diari Oficial de la Generalitat de Catalunya, no 5357 (2009).

(14) Cataluña, España: "Decreto 183/2010, de 23 de noviembre, de establecimientos de alojamiento turístico". Diari Oficial de la Generalitat de Catalunya, no 5764 (2010).

(15) Cataluña, España: "Orden de 11 de julio de 1986, por la que se establecen los requisitos para la instalación y el funcionamiento de los campings". Diari Oficial de la Generalitat de Catalunya, $\mathrm{n}^{\circ} 717$ (1986).

(16) España: "Real Decreto de 12 de julio, de ordenanzas técnicas y normas constructivas para viviendas de renta limitada". Boletín Oficial del Estado, no 197 (1955).

(17) España: "Orden Ministerial de 20 de mayo, adaptación de las ordenanzas técnicas y normas constructivas de viviendas de protección oficial". Boletín Oficial del Estado, n 123 (1969).

(18) España: "Orden de 24 de noviembre de 1976, por la que se aprueban las normas técnicas de diseño y calidad de las viviendas sociales". Boletín Oficial del Estado, n 296 (1976).

(19) España: "Orden ministerial de 21 de febrero de 1981, por la que se modifican las ordenanzas técnicas y normas constructivas novena, undécima, decimotercera, decimoséptima y trigésimo cuarta aprobadas por la Orden de 20 de mayo de 1969". Boletín Oficial del Estado, no 53 (1981).

Artículo redactado en el marco de una investigación financiada con fondos procedentes del programa FI de la Generalitat de Catalunya (2009-2012).
(20) Ministerio de Fomento: Políticas de intervención en edificios existentes. La adecuación del CTE a la rehabilitación. Construmat 2011. Barcelona, 2011. 